\title{
Analog Self-Interference Cancellation with Automatic Gain Control for Full-Duplex Transceivers
}

\author{
Visa Tapio, Marko Sonkki and Markku Juntti \\ Centre for Wireless Communications \\ University of Oulu, Finland \\ Email: firstname.lastname@oulu.fi
}

\begin{abstract}
The main challenge in full-duplex transceiver design is the self-interference (SI). Analog SI isolation is performed at radio frequency (RF) by using an antenna design based on the characteristic modes theory and using active cancelation principle. Two different structures based on using a vector downconverter and a complex multiplier are used for analog baseband SI cancellation. Cancellers are tuned using a automatic gain control (AGC) enhanced variable-step steepest descent algorithm while transmitting a data signal to a distant node in half-duplex mode. Simulations show that the inclusion of AGC into the tuning process speeds up the convergence significantly.
\end{abstract}

Index terms - Full-duplex, self-interference, adaptive cancellation

\section{INTRODUCTION}

Full-duplex (FD) transceivers can transmit and receive simultaneously at the same carrier frequency offering the potential to double the spectral efficiency, to reduce air interface delays, and to facilitate improved collision detection and avoidance mechanisms in content based networks. Other benefits as well as challenges in the FD technology development have been reviewed in, e.g., [1], [2], [3].

The main problem in the FD transceiver design is the selfinterference (SI), i.e., the leakage of the transmit signal to the device's own receiver. Depending on the system, the SI cancellation requirement can be well over $100 \mathrm{~dB}$. In order to achieve such a high isolation levels, the SI cancellation must be done at different stages. Reviews on different SI cancellation techniques can be found, e.g., in [4], [5], [6]. In this paper, the self-interference cancellation is performed with three techniques: antenna isolation, active cancellation at radio frequency (RF) and analog cancellation at the baseband. Since the attenuation and phase settings of the active cancellation can change during the operation, they must be tunable. The usage of a gradient descent algorithm for the tuning has been proposed in [7]. Designs reported in [8], [9] also use iterative algorithms to adjust the phase and attenuation in the feed forward paths of the analog SI cancellers. The tuning of the SI cancellation in [7], [8], [9] is done prior the full-duplex communication. Designs in [8], [9] are front end-designs where the tuning algorithms are run on a specific control unit and an additional analog-to-digital (AD) conversion is needed for the SI cancelation. In our design, all the processing is performed at the baseband processing unit, hence no additional
$\mathrm{AD}$ converters are needed for the SI cancellation. The tuning of the RF and baseband cancellation circuitry is done in the half-duplex (HD) mode using the transmitted data signal without the need to use a specific tuning signal, i.e., the data transmission can be continuous. Tuning is performed with the steepest descent algorithm. Automatic gain control (AGC) is used to scale the input signals of AD converters to fully utilize the dynamic range of the converters. Simulations show that this speeds up the convergence of the tuning considerably. After the tuning is completed, the system can be switched to the FD mode.

The SI channel consists of the leakage through the antenna and the reflections from the operating environment of a FD transceiver. Although there are some published measurements of a SI channel [10], [11] there are no widely accepted models for the SI channel. A SI channel modeling based on a ray tracing approach is proposed herein in Section III. It allows a relatively simple way to model a multipath SI channel.

Main contributions considered are the inclusion of the AGC in the tuning process, introduction of the analog complex multiplier based baseband cancellation and the SI channel modeling using a ray tracing approach. The rest of the paper is organized as follows. The transceiver model and the SI cancellation are described in Section II, SI channel model is presented in Section III and numerical results are presented in Section IV. Finally, conclusions are presented in Section V.

\section{Transceiver AND SELF-InTERFEREnCE CANCELlation}

A block diagram of a full duplex transceiver is shown in Fig. 1. Four different techniques for the SI cancellation are included in the architecture: isolation provided by the antenna, SI cancellation at RF, SI cancellation at analog baseband and digital cancellation. Antenna isolation, RF cancellation and analog baseband cancellation are considered. Antenna design is based on the characteristic modes theory [12], [13]. The same antenna model was used also in [14], [15]. The RF canceller is is shown in Fig. 2. It consists of a FIR filter type structure where the phase and gain values of each tap can be tuned. The delay $\tau$ equals the sample time of the analog-to-digital (AD) converters. Two different structures for the analog baseband SI cancellation are presented in 


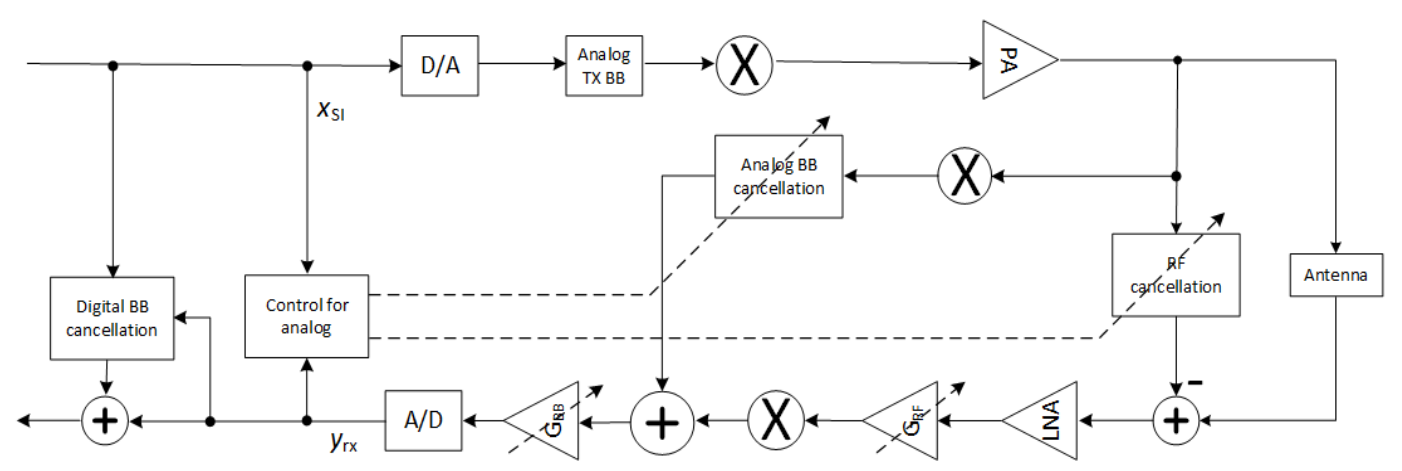

Fig. 1. FD transceiver architecture.

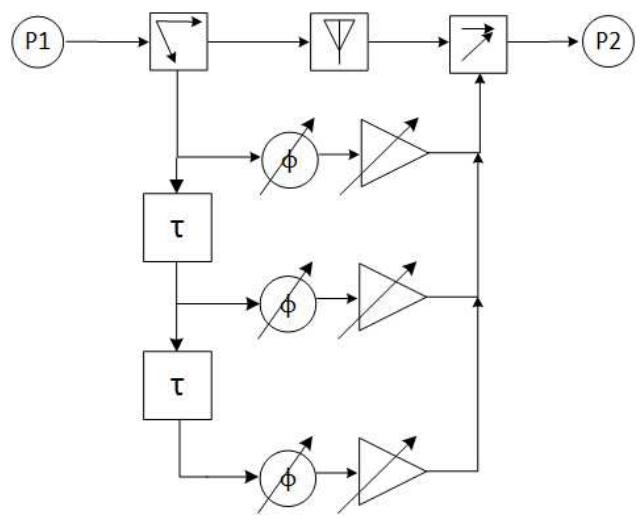

Fig. 2. Antenna and RF SI cancellation.

Fig. 3. The first one uses vector down converter to take into account the phase shift caused by the SI channel, the other uses analog complex multiplier to compensate for the phase rotation. The vector down converter based canceller requires the phase to be estimated. Since its output is a nonlinear function of the phase, its tuning is more complicated than the tuning of the complex multiplier. In the complex multiplier, the output is a linear function of the gain values that need to be estimated. Only one-tap baseband cancellers are considered. The complex multiplier based canceller can be extended to multi-tap structure for multipath SI channel cases but that requires the inclusion of tunable delay elements into the canceller and the usage of an algorithm to estimate the delay and gain values of an multipath channel.

Since changes in the environment can cause the SI channel to change, the SI cancellers must be tunable. The tuning of the analog cancellers is done with a transmitted data signal in a half duplex mode, i.e, no FD specific training signals for the tuning are needed. Both the RF and baseband cancellers are tuned using a steepest descent algorithm. At each iteration step, a complex coefficient vector $\mathbf{w}(k)$ is calculated as

$$
\mathbf{w}(k)=\mathbf{w}(k-1)-\frac{\mu_{i}}{M P_{\mathrm{x}}} \sum_{n=1}^{M} y_{\mathrm{rx}}(n) \mathbf{x}_{\mathrm{SI}}^{*}(n),
$$

where $k$ is the iteration index, $M$ is the number of samples per iteration, $P_{\mathrm{x}}$ is the power of the SI signal $x_{\mathrm{SI}}, y_{\mathrm{rx}}$ is the received signal and $\mu_{i}$ is the step size of the algorithm. The numerical values $\mu_{i}$ depend on the properties of the signal as well as on the properties of the transceiver, especially the gain of the receiver, and they have been selected by simulations to allow fast convergence at the beginning and good accuracy at the end of the tuning. Vector $\mathbf{x}_{\mathrm{SI}}$ consists of samples $x_{\mathrm{SI}}(n), x_{\mathrm{SI}}(n-$ 1) $\cdots x_{\text {SI }}(n-N)$ for a $N$ length SI canceller.

Since the SI level is reduced during the tuning, the signal at the input of $\mathrm{AD}$ converters is reduced. In order to utilize the full dynamic range of the AD converters during the tuning, AGC is added to the model. In the case of the RF canceller the gain of the amplifier $G_{R F}$ in Fig. 1 is controlled using Algorithm 1 . In baseband cancellation case the $G_{B B}$ is controlled with the same algorithm.

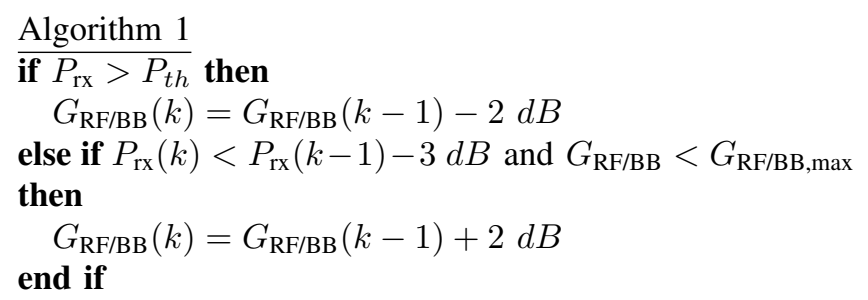

$P_{t h}$ is a threshold power for preventing ADC overload. It was shown in [14] that using a variable step size in the SD algorithm can improve the convergence rate. In this case, the step is chosen based on the AGC value using Algorithm 2.

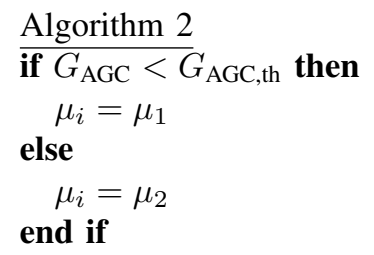

\section{SElF-Interference ChanNEl Model}

The SI channel consists of the direct coupling through the antenna and reflections from the environment. Reflections are modeled using a ray tracing approach. The attenuation of a reflection path $i$ is

$$
L_{i}=L_{\mathrm{free}}^{i}+L_{\mathrm{R}}^{i}-G_{\mathrm{tx}}^{i}-G_{\mathrm{rx}}^{i},
$$



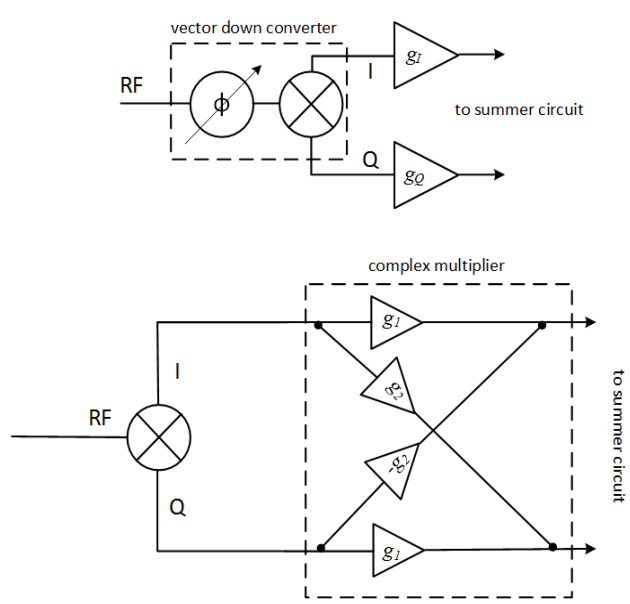

Fig. 3. Analog baseband SI cancellation.

where $L_{\text {free }}^{i}$ is the free space loss of the $i^{\text {th }}$ path

$$
L_{\text {free }}^{i}=20 \log _{10}\left(2 d_{i}\right)+20 \log _{10} f+\log _{10} \frac{4 \pi}{c},
$$

$d_{i}$ is the distance in meters between the transceiver and reflecting surface, $f$ is the frequency in herz and $c$ is the speed of electromagnetic radiation. The loss component caused by the reflection is

$$
L_{R_{i}}=20 \log _{10} R_{i},
$$

where the reflection coefficient $R_{i}$ is [16], [17]

$$
R_{i}=\frac{\sin \theta_{i}-Z_{i}}{\sin \theta_{i}+Z_{i}}
$$

and [16], [17]

$$
Z_{i}= \begin{cases}\sqrt{\frac{1}{\varepsilon_{r_{i}}^{2}}\left(1-\cos \theta_{i}\right)} & \text { (vertical polarization) } \\ \sqrt{\epsilon_{r_{i}}-\cos ^{2} \theta_{i}} & \text { (horizontal polarization). }\end{cases}
$$

$\varepsilon_{r_{i}}$ is the relative permittivity of the $i^{\text {th }}$ reflecting surface and $\theta_{i}$ is the grazing angle of $i^{t h}$ reflection. $G_{\mathrm{tx}}^{i}$ and $G_{\mathrm{rx}}^{i}$ in (2) are the gains of the antenna in the transmit and reception direction of the reflections. Since the $i^{t h}$ reflection path is assumed to include only one reflection, these gains are equal but the ray tracing approach can also be extended to include multiple reflections in a single multipath component. The relative permittivity of some of the construction materials are listed in Table I. In the same table reflection losses with some grazing angles are also given.

\section{NUMERICAL EXAMPLES}

The signal used in the simulations is an orthogonal frequency-division multiplexing (OFDM) signal with 48 data sub-carriers and 4 pilot sub-carriers at $3.5 \mathrm{GHz}$ center frequency. Data sub-carriers are modulated using 16 level quadrature amplitude modulation (16-QAM). The bandwidth of the signal is $20 \mathrm{MHz}$. The antenna is modelled using an electromagnetic simulation tool (CST Microwave Studio). Simulated S-parameters are brought to the system model as a S-parameter file. The isolation of the antenna over the signal bandwidth is $61 \mathrm{~dB}$. The FD transceiver including the analog SI cancllers and AD converters is modelled using the Advanced Design System (ADS). The maximum values of the integral (INL) and differential (DNL) non-linearity to least significant bit of the AD converters are 5.0 and 0.7 , respectively. The phase and amplitude imbalance of the transceivers are $0.5^{\circ}$ and $0.1 \mathrm{~dB}$, respectively, and the phase noise dat is taken from [18], [14].

\section{Case 1: No Reflections from the Environment}

Firstly, the SI consists of only the signal leaking through the antenna from the transmitter to the receiver. This means that the SI channel consists of the transmitter chain, antenna and receiver chain. The used antenna provides $61 \mathrm{~dB}$ of isolation over $20 \mathrm{MHz}$ bandwidth and RF and analog baseband circuitry can be used to improve the isolation.

\section{Case 2: Multipath SI channel}

Secondly, the SI consists of the signal leaking through the antenna and signals reflecting back from the objects or the surfaces in the surrounding environment of the FD transceiver. The antenna cannot attenuate the signal components reflecting the surface. Hence, the isolation provided by the antenna is lower than in the Case 1 . The SI channel has 3 paths caused by the reflections in addition to the leakage through the antenna. The reflections are modeled using the ray tracing approach described in Section III. The reflecting surfaces are assumed to be at the distances of $0.5 \mathrm{~m}, 1 \mathrm{~m}$ and $3 \mathrm{~m}$ from the transmitter. The reflection loss is assumed to be $8 \mathrm{~dB}$, and antenna gain is $3 \mathrm{~dB}$. This gives the delay attenuation pairs pairs $(3.33 \mathrm{~ns}, 45 \mathrm{~dB}),(6.67 \mathrm{~ns}, 50 \mathrm{~dB})$ and $(20 \mathrm{~ns}, 55 \mathrm{~dB})$ for the paths. In addition, a 180 degree phase shift is assumed for all the reflections. With these reflections the antenna isolation is decreased to $49 \mathrm{~dB}$.

\section{A. RF Cancellation}

The performance of a 1-tap RF canceller is shown in Fig. 4. The vertical axis shows the amount of cancellation in decibels $(\mathrm{dB})$ provided by the canceller and the horizontal axis shows the number of iterations needed for the tuning ( $k$ in Algorithms 1 and 2). The SIC_RF_NO_ref curve shows the performance in the Case 1 and the SIC_RF_nref curve the Case 2. The total isolation after the convergence in the Case 1 is $25+61 \mathrm{~dB}$ and in the Case $218+49 \mathrm{~dB}$.

The SI cancellation performance of a 3-tap canceller in Case 2 is shown in Fig. 5. The SIC_RFn1 curve (blue line with circles) and the SIC_RFn curve (red) show the performance when the step size $\mu$ of the SD algorithm is $10 \cdot 10^{-3}$ and $15 \cdot 10^{-3}$, respectively. As can be seen, the step size can be used to control the convergence rate of the tuning but the tuning is slow. In both of these cases the gains $G_{R F}$ and $G_{B B}$ chain has been constant and it has been set so that the AD converters do not limit the peaks of their input signals. The black SIC_RFn2 curve with 'x' markers shows the performance when the AGC has been used to control the gain $G_{R F}$ and the step size is selected based on the gain value. The usage of the AGC and 
TABLE I

REFLECTION LOSSES CAUSED BY SOME CONSTRUCTION MATERIALS

\begin{tabular}{|c|c|c|c|c|c|c|c|c|c|c|c|}
\hline \multirow{2}{*}{ Material } & \multirow[b]{2}{*}{$\varepsilon_{r}$} & \multicolumn{5}{|c|}{ Vertical polarization } & \multicolumn{5}{|c|}{ Horizontal polarization } \\
\hline & & $30^{\circ}$ & $45^{\circ}$ & $60^{\circ}$ & $75^{\circ}$ & $90^{\circ}$ & $30^{\circ}$ & $45^{\circ}$ & $60^{\circ}$ & $75^{\circ}$ & $90^{\circ}$ \\
\hline Glass window & 6.5 & -16.4 & -10.3 & -8.3 & -7.4 & -7.2 & -3.7 & -5.2 & -6.3 & -7.0 & -7.1 \\
\hline Concrete (dry) & 4.5 & -22.5 & -12.8 & -10.2 & -9.2 & -8.9 & -4.6 & -6.4 & -7.8 & -8.6 & -8.9 \\
\hline Chipboard & 2 & -25.1 & -22.9 & -17.5 & -15.8 & -15.3 & -8.4 & -11.4 & -13.6 & -14.9 & -15.3 \\
\hline
\end{tabular}

variable step SD speeds up convergence substantially. After the convergence the RF canceller provides $34 \mathrm{~dB}$ of additional SI attenuation $(34+49 \mathrm{~dB}$ in total). RF cancellers in [8], [9] provide about (30) $\mathrm{dB}$ of cancellation. However, it should be taken into account that the results in [8], [9] are from real measurements. Simulation results shows that it is possible to achieve good SI cancellation performance using the data signal for the tuning and without the need for independent front end designs where tuning are run on a specific control unit with additional AD converters.

\section{B. Analog Baseband Cancellation}

The performances of the both analog baseband cancellers in the Case 1 are shown in Fig. 6. The SIC_I and SIC_Q curves show the convergence and SI cancellation at the I and $\mathrm{Q}$ branches of the receiver before the $\mathrm{AD}$ converters when the vector down converter followed by two tunable amplifiers are used. At the beginning of the tuning, the amplifier gains are set to zero. This means that the canceller provides no additional cancellation at the beginning. The phase shift of the vector down converter is set to the phase shift of the antenna at the center frequency. This value is taken from the known S-parameter model and it is not changed during the tuning. Amplifiers at the I and Q branches of the canceller are tuned independently using the received I and Q signals after the AD converters. Tuning is done using the SD algorithm with constant step size.

SIC_BB_I and SIC_BB_Q curves show the performance of the analog complex multiplier based SI cancellation. The gains of the canceller are set to zero at the beginning of the tuning and the tuning is done using the received complex baseband signal after the AD converters. Tuning is done using the SD algorithm with constant step size and AGC is used to control the gain of amplifiers $G_{B B}$ following the SI canceller. As can be seen, both structures give the same performance of after the tuning but the complex multiplier based canceller has converged much faster than the vector down converter based structure. The curves in the Fig. 6 show the additional SI attenuation provided by the baseband cancellers. The total SI cancellation is the sum of the antenna isolation and the additional cancellation shown in the figure $(=30+61 \mathrm{~dB})$.

Results of analog baseband cancellation have been reported in [19], [20]. Measured baseband SI cancellation performance in those papers has been about $20 \mathrm{~dB}$. A vector down converter is used in [19] to rotate the phase of the SI signal used for the cancellation. The signal at the input is first attenuated at $\mathrm{RF}$ and then down converted using a vector down converter whereas here the level of the signals at the I and Q branches are independently controlled after the down conversion. In [20] the baseband canceller uses a 14-tap filter for the analog cancellation. No details are given about the tuning of the circuit but since its performance is at the same level as in [19] and lower than reported in this paper, it is possible that the tuning has converged into the state where only one tap is contributing to the cancellation.

The complex multiplier based canceller was tested also in the Case 2. The performance in this case is presented with curves SIC_BB_I1 and SIC_BB_Q1. As can be seen the baseband canceller gives $20 \mathrm{~dB}$ of additional cancellation to the antenna isolation (total isolation $49+20 \mathrm{~dB}$ ).

\section{CONCLUSIONS}

A SI channel model consisting of the leakage path through the antenna and reflections from the environment was introduced. Reflection paths were modeled using a ray tracing approach, where the reflection losses are calculated based on the electrical properties of the reflecting surfaces. The performances of RF and analog baseband SI cancellation were evaluated with simulations. The inclusion of the AGC into the tuning process speeds up the convergence significantly. In the simulated multipath SI channel case, the RF canceller provides $30 \mathrm{~dB}$ of additional cancellation when compared with the case where only the antenna is providing isolation between the transmitter and receiver.

Two structures for 1-tap analog baseband SI cancellation were compared. The first one uses vector down-converter to compensate for the phase shift of the SI channel, the second one utilizes complex multiplier structure. They both provide about the same isolation after the tuning but the complex multiplier based solution is easier to tune and it converges very rapidly. When there are no reflections from the environment the baseband cancellation gives better performance than the 1-tap RF canceller and it is also able to provide additional isolation in the multipath case.

\section{ACKNOWLEDGMENT}

The research has been partially funded by Tekes - the Finnish Funding Agency for Innovation, Nokia Oyj, Esju Oy and CoreHW

\section{REFERENCES}

[1] S. Hong, J. Brand, J. I. Choi, M. Jain, J. Mehlman, S. Katti, and P. Levis, "Applications of self-interference cancellation in 5g and beyond," IEEE Communications Magazine, vol. 52, no. 2, pp. 114-121, February 2014

[2] A. Sabharwal, P. Schniter, D. Guo, D. W. Bliss, S. Rangarajan, and R. Wichman, "In-band full-duplex wireless: Challenges and opportunities," IEEE Journal on Selected Areas in Communications, vol. 32, no. 9, pp. 1637-1652, Sept 2014. 


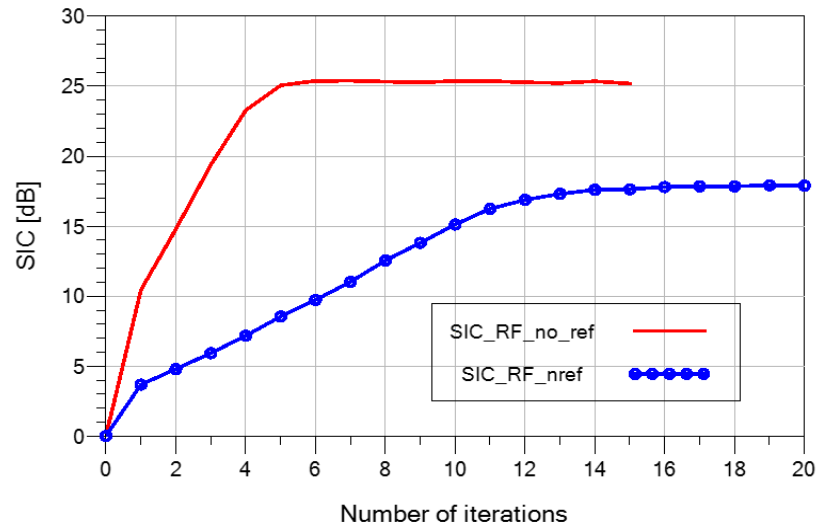

Fig. 4. Performance of the 1-tap RF SIC.

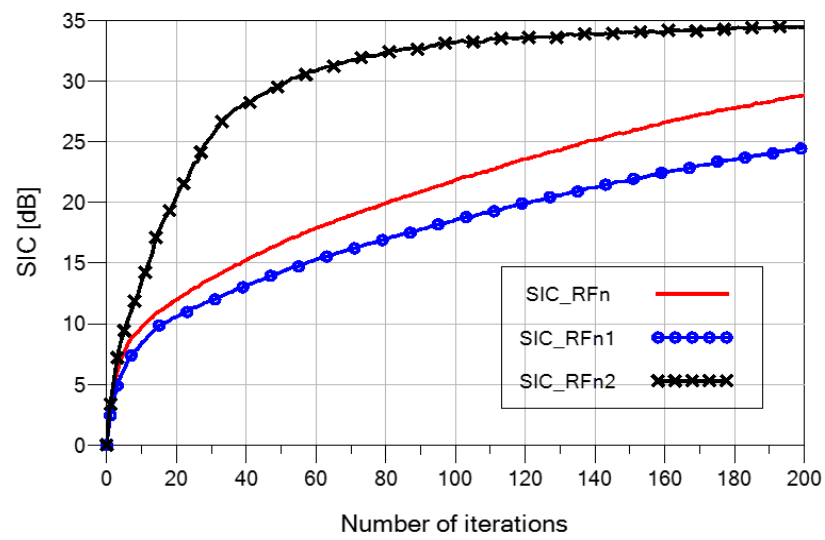

Fig. 5. Performance of the 3-tap RF SIC.

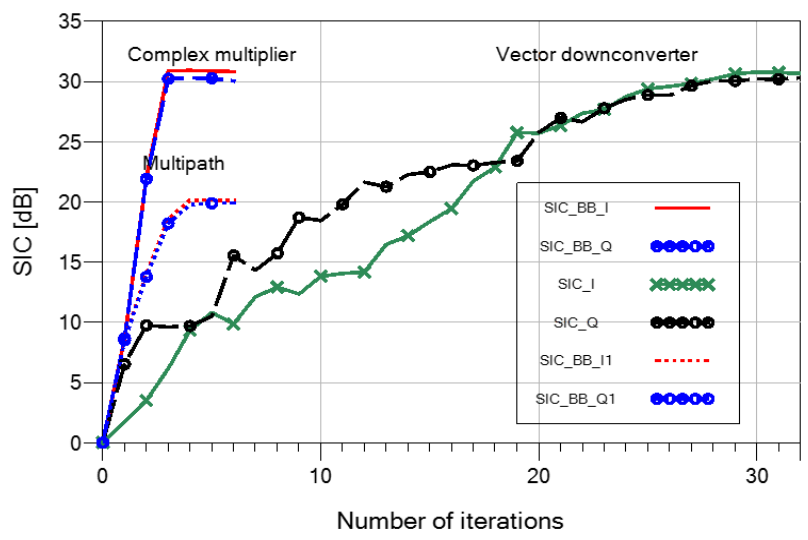

Fig. 6. Performance of the analog baseband SIC.
[3] K. Rikkinen, M. Juntti, V.Tapio, A. Pouttu, B. Debaillie, C. Lavin, and M. Ghoraishi, "Full duplexing," in 5G Wireless Technologies, A. Alexiou, Ed., chapter 8. IET Publishing, ISBN: 978-1-78561-061-5, 2017.

[4] Z. Zhang, X. Chai, K. Long, A. V. Vasilakos, and L. Hanzo, "Full duplex techniques for $5 \mathrm{~g}$ networks: self-interference cancellation, protocol design, and relay selection," IEEE Communications Magazine, vol. 53, no. 5, pp. 128-137, May 2015.

[5] M. Heino, D. Korpi, T. Huusari, E. Antonio-Rodriguez, S. Venkatasubramanian, T. Riihonen, L. Anttila, C. Icheln, K. Haneda, R. Wichman, and M. Valkama, "Recent advances in antenna design and interference cancellation algorithms for in-band full duplex relays," IEEE Communications Magazine, vol. 53, no. 5, pp. 91-101, May 2015.

[6] B. Debaillie, D. J. van den Broek, C. Lavn, B. van Liempd, E. A. M. Klumperink, C. Palacios, J. Craninckx, B. Nauta, and A. Prssinen, "Analog/rf solutions enabling compact full-duplex radios," IEEE Journal on Selected Areas in Communications, vol. 32, no. 9, pp. 1662-1673, Sept 2014.

[7] M. Jain and et al., "Practical, real-time, full duplex wireless," in Proceedings of the 17th Annual International Conference on Mobile Computing and Networking (MobiCom'11), New York, NY, USA, 2011, pp. 301-312, ACM.

[8] T. Huusari, Y. S. Choi, P. Liikkanen, D. Korpi, S. Talwar, and M. Valkama, "Wideband self-adaptive rf cancellation circuit for fullduplex radio: Operating principle and measurements," in 2015 IEEE 81st Vehicular Technology Conference (VTC Spring), May 2015, pp. $1-7$.

[9] K. E. Kolodziej, J. G. McMichael, and B. T. Perry, "Multitap rf canceller for in-band full-duplex wireless communications," IEEE Transactions on Wireless Communications, vol. 15, no. 6, pp. 4321-4334, June 2016.

[10] Kun Wang, Ruonan Zhang, Zhimeng Zhong, Xiaomei Zhang, and Xiaoyan Pang, "Measurement of self-interference channels for full-duplex relay in an urban scenario," in 2017 IEEE International Conference on Communications Workshops (ICC Workshops), 2017, pp. 1153-1158.

[11] A. Sethi, V. Tapio, and M. Juntti, "Self-interference channel for full duplex transceivers," in 2014 IEEE Wireless Communications and Networking Conference (WCNC), April 2014, pp. 781-785.

[12] R. Garbacz and R. Turpin, "A generalized expansion for radiated and scattered fields," IEEE Transactions on Antennas and Propagation, vol. 19, no. 3, pp. 348-358, May 1971.

[13] R. Harrington and J. Mautz, "Theory of characteristic modes for conducting bodies," IEEE Transactions on Antennas and Propagation, vol. 19, no. 5, pp. 622-628, Sep 1971.

[14] V. Tapio, M. Juntti, A. Prssinen, and K. Rikkinen, "Real time adaptive rf and digital self-interference cancellation for full-duplex transceivers," in 2016 50th Asilomar Conference on Signals, Systems and Computers, Nov 2016, pp. 1558-1562.

[15] V. Tapio, H. Alves, and M. Juntti, "Joint analog and digital selfinterference cancellation and full-duplex system performance," in 2017 IEEE International Conference on Acoustics, Speech and Signal Processing (ICASSP), March 2017, pp. 6553-6557.

[16] A. Goldsmith, Wireless Communications, Cambridge University Press, 2005.

[17] "Reflection from the surface of the earth," Report 1008-1, International Telecommunication Union (ITU).

[18] "MAX/2828/2829 Single-/Dual-Band 802.11 a/b/g World-Band Transceiver ICs," Data sheet, Maxim Integrated Products, Inc.

[19] D. J. van den Broek, E. A. M. Klumperink, and B. Nauta, "19.2 a self-interference-cancelling receiver for in-band full-duplex wireless with low distortion under cancellation of strong tx leakage," in 2015 IEEE International Solid-State Circuits Conference - (ISSCC) Digest of Technical Papers, Feb 2015, pp. 1-3.

[20] T. Zhang, A. Najafi, C. Su, and J. C. Rudell, "18.1 a 1.7-to-2.2ghz fullduplex transceiver system with $>50 \mathrm{db}$ self-interference cancellation over 42mhz bandwidth," in 2017 IEEE International Solid-State Circuits Conference (ISSCC), Feb 2017, pp. 314-315. 